\title{
Kritik am Beitrag «Evolution der Krankenrolle - eine These»
}

Rolf Tschannen

1 Knecht T: Evolution der Krankenrolle - eine These. Schweiz Ärztezeitung. 2010;91(46):1838-41.

Korrespondenz:

Dr. med. Rudolf Tschannen Thuraustrasse 12 CH-9500 Wil

psirolf@swissonline.ch
Nicht ganz ohne Absicht, dabei aber durchaus auch etwas augenzwinkernd, sucht der Schreibende mit vorliegendem Aufsatztitel Anschluss an eine Reihe berühmterer philosophischer Abhandlungen zu knüpfen. Die Motivation dazu entspringt sowohl der Auseinandersetzung mit der Darstellung der Krankenrolle in der Arbeit von Thomas Knecht [1] als auch einem Rückblick auf eigene dreissigjährige psychiatrische und auch gutachterliche Praxis.

Knecht entwickelt seinen Grundsatz zum Verständnis der Krankenrolle als darwinistischen Anpassungsvorgang im gesamten Leidens- und Hilfssystem schlüssig und einleuchtend. Dabei verzichtet er auf populistische Auslegungen seiner These wie die geschlechts- und kulturspezifischen Unterschiede im Erleben und Ausleben der Betroffenen oder die naheliegende Darstellung historischer Zusammenhänge wie derjenigen zwischen Unfallversicherung und Industrialisierung oder Militärversicherung und der Einführung der allgemeinen Wehrpflicht. Seiner Darstellung folgend lässt sich aus dem co-evolutionären Prozess schlüssig die Notwendigkeit auch in Zukunft stets wiederholter Revisionen des AHV-, IV- und Krankenversicherungsrechtes ableiten. Dass die systemische Kommunikation zwischen Hilfsbedürftigen und Helfern zusammengefasst einem darwinistischen Optimierungsprozess gehorcht, hat Knecht somit überzeugend dargestellt und empfindet der Schreibende als überdenkenswerte Aussage. So weit ich das erkennen kann, ist jedoch in der noch kritisch zu betrachtenden These der Begriff der «Krankenrolle» nicht näher beleuchtet worden, und das möchte dieser Aufsatz in einigen Grundzügen nachholen:

Wie ist der Begriff «Krankenrolle» definiert? Angelehnt an den Duden, handelt es sich bei der «Rolle» einerseits um die von einem Schauspieler zu verkörpernde Gestalt, um eine Darstellung, ein Schau-Spiel eben, und andererseits um das erwartete Verhalten innerhalb der Gesellschaft. Offenbar schwebte Knecht vor allem diese zweite Bedeutung vor, die in der Tat ja bereits in der Definition das systemische Element enthält. Allerdings schimmert bei ihm verschiedentlich auch die erste Bedeutung durch, etwa dort, wo der Autor auf ungerechtfertigte, unechte, kurz irgendwie «gespielte» Hilfeäusserungen verweist. Darüber hinaus ist an diesem Begriff jedoch vor allem ein Gesichtspunkt von grösster Bedeutung: Offenbar handelt es sich bei der «Krankenrolle» um einen Ausdruck aus der Welt unserer Vorstellungen, des Konstruierten, des mit dem kognitiven Apparat Wahrgenommenen bzw. Ausgedrückten, der Kausalität gehorchenden Erlebens und nicht um «ein Ding an sich».

Schmerz und Leiden jedoch lassen sich in ihrem jeweiligen Erlebens- und Auslebenscharakter nicht nur oder überwiegend im Sinne einer konstruktivistisch verstandenen darwinistischen Auslese- oder Anpassungsstrategie begreifen. Und zwar handelt es sich dabei auch um sehr unmittelbare, letztlich zeitlose, damit akausale, anthropologische Phänomene

\section{Eine «Krankenrolle» ist etwas grundsätzlich anderes als ein krankes Lebewesen.}

beispielsweise orientierenden Charakters: Vorzüglich auf diese Weise werden wir nämlich jeweils ungewollt und wahrlich unmittelbar schmerzhaft mit den Grenzen von Menschsein und Gesundheit konfrontiert. Das geschieht erst sekundär auf einer Ebene kognitiver Konstruktion, es beinhaltet primär vielmehr die unmittelbare Erfahrung einer überraschenden, recht eigentlich erschütternden, Wendung des LebensWillens im Sinne etwa von Schopenhauer. Nun hat Knecht, so weit ich das überblicke, den Begriff der "Krankenrolle« in seiner zeichenhaften, semiotischen Bedeutung zwar tatsächlich nirgends mit demjenigen der Krankheit als etwas Ursprünglichem, keinerlei kausalen Prinzipien gehorchendem Erlebnis, durcheinandergeworfen, so dass hier keine Kritik angebracht ist. Dagegen kann man ihm den Vorwurf nicht ersparen, dass er gerade diese Unterschiede nicht aufgezeigt und damit auf die Grenzen der Erklärungsfähigkeit seines Themas nicht hingewiesen sowie Missverständnissen Tür und Tor geöffnet hat, weil eben eine «Krankenrolle» etwas grundsätzlich anderes ist als ein krankes Lebewesen.

Vielleicht ist es das Überhandnehmen dieser Welt der Vorstellung, des Scheins, der hinduistischen Maya, die zum hiesigen allmählichen Verlust der Demut im gesellschaftlichen und auch medizinischen Diskurs geführt hat. Stattdessen findet nämlich der von Illusionen genährte Machbarkeitswahn auch im Gesundheitswesen ungesunden, hypertrophierenden Nährboden: entsprechend die neueste Formulierung aus 
einem Lokalblatt: «Tod als Chance»! Nun hat die «Welt als Vorstellung» auch ihre unbestreitbaren Vorteile, und einer der nicht unwesentlichsten dürfte deren Messbarkeit und Kausalität sowie damit der Zugang zu einem rationaleren Diskurs darstellen. Dass sich gerade die Psychiatrie dessen im von mir überblickbaren Zeitraum in deutlich vermehrtem Ausmass bedient, gereicht in mancherlei Hinsicht auch zu deren Vorteil. Ich bedauere keineswegs die Tatsache der verstärkt naturwissenschaftlichen Ausrichtung, sondern vielmehr die dabei fehlende SelbstReflexion und damit Einseitigkeit: Konnten wir vor einem Vierteljahrhundert noch davon sprechen, einem leidenden Menschen therapeutisch zu begegnen, sich in sein Dasein einzulassen, so geht es hier und jetzt um «störungsspezifische» Therapien. Mit diesem Kniff ist es unserer Fachdisziplin immerhin gelungen, unsere eigene «darwinistische Attraktivität» weg von der mitmenschlichen Solidarität (vgl. etwa Guggenbühl, Macht als Gefahr beim Helfer [2]) hin zu eben am liebsten von Experten maximalisierbar Machbarem zu tun, was im Aufsatz von Knecht zwar zu Recht angeprangert wird, dem diese Sichtweise jedoch insgesamt Vorschub leistet.

\section{Die Verantwortung sich und anderen gegenüber wird nicht wahrgenommen; das besorgt dann die Rechts- schutzversicherung.}

2 Guggenbühl A. Macht als Gefahr beim Helfer. Basel: Karger; 1987.

3 Studer K. Aktualitäten. Schweizer Archiv für Neurologie und Psychiatrie 2010;161(2):78

4 Safranski R. Schopenhauer und die wilden Jahre der Philosophie. München: Hanser; 2010. S. 179-80. rungen schliesslich als Ausdruck einer «Hypersolidarität» gesehen. Wiederum dem Duden folgend, müsste man darunter ein übermässiges, unbedingtes Zusammenhalten mit jemandem aufgrund gleicher Anschauungen und Ziele oder eine Unterstützung, die sich auf das Zusammengehörigkeitsgefühl und das Eintreten füreinander gründet, verstehen. Zentral wäre demgemäss hier letztlich ein «übermässiges» Mitgefühl.

Nicht nur ich sehe aber für ein solches, echtes Mitgefühl in unserer globalisierten, mindestens sehr stark bevölkerten und ausgebeuteten Welt keine Zeichen, vielmehr für das Übergewicht egoistischer darwinistischer Überlebensstrategien. Dazu werden vielerorts verschiedene gewichtige Gründe angeführt, wonach wir in unserer Ära der Versicherungen, Krankenkassen, Zertifizierungen und des Controllings gar nicht in einem hypersolidarischen, sondern vielmehr in einem entsolidarisierten Zeitalter leben. Heute sei nämlich persönliche Verantwortung sich und anderen (Mitmenschen, leidend oder nicht, überhaupt der Mitwelt) gegenüber im Abnehmen begriffen und würde stattdessen delegiert; dies nicht nur an zahlreiche (Hilfs-)Organisationen, sogar auch an psychologische Tests zum Entlarven von «Schein-Invaliden» beispielsweise, womit uns hier Validierbarkeit von der Selbstreflexion entlastet. So schreibt Safranski [4]: «Die weitere Geschichte der Allianz der bürgerlichen Gesellschaft mit der Welt der Moral ist bekannt, sie verläuft nach der Devise: Vertrauen ist gut, Kontrolle ist besser. Der Bedarf an Gewissensverinnerlichung hat vollends in unserem Jahrhundert dramatisch abgenommen: Es hat staatliche Ermunterung zur Gewissenlosigkeit grössten Stils gegeben, gleichzeitig sind die Kontrollnetze von oben noch engmaschiger geworden, und aus dem neu entdeckten psychischen Untergrund quillt eine ganze Kultur der Ausrede, der Unbelangbarkeit - so dass dem Gewissen nur noch wenig zu tun bleibt, weshalb es auch wieder aufs vorneuzeitliche Niveau herunterkümmert und seinen Ablasshandel mit Strafzetteln und Krankenscheinen betreibt.»

Richtig ist, dass wir aus der allgegenwärtigen, durchaus selbstbezogenen und somit keineswegs «hypersolidarischen» Angst geradezu einem Versicherungswahn verfallen sind (z. B. die »Armee als [günstigste] Versicherung») und im Rahmen des überschätzten Fortschrittsglaubens schlechthin alles gegen alles versichern möchten, gerade weil die Verantwortung sich und anderen gegenüber nicht wahrgenommen wird; das besorgt dann die Rechtsschutzversicherung. Gleichartig werden unerfüllbare Sicherheitshoffnungen gegebenenfalls über einen Anruf bei Mike Shiva oder eine Konsultation von Madame Etoile zu stillen versucht.

\section{Ein entsolidarisiertes Zeitalter}

In der Arbeit von Knecht werden die übermässig zugenommenen Invaliditätsleistungen und -forde- 